\title{
Mucopolysaccharidosis Type I
}

National Cancer Institute

\section{Source}

National Cancer Institute. Mucopolysaccharidosis Type I. NCI Thesaurus. Code C85053.

The most common type of mucopolysaccharidosis. It is inherited in an autosomal recessive pattern. It comprises a group of lysosomal storage diseases which includes the most severe form (Hurler syndrome) and the mildest form (Scheie syndrome). 